\title{
Exploring student conceptual resources about heat and temperature
}

\author{
Yohannes M. Abraham \\ Department of Biology, Seattle Pacific University, 3307 Third Ave W, Seattle, WA, USA, 98119-1997 \\ Mikayla Valentin \\ Department of Physics, Seattle Pacific University, 3307 Third Ave W, Seattle, WA, USA, 98119-1997 \\ Brynna Hansen \\ Department of Biology, Seattle Pacific University, 3307 Third Ave W, Seattle, WA, USA, 98119-1997 \\ Lauren C. Bauman \\ Department of Physics, University of Washington, Box 351560, Seattle, WA, USA, 98195-15603 \\ Amy D. Robertson \\ Department of Physics, Seattle Pacific University, 3307 Third Ave W, Seattle, WA, USA, 98119-1997
}

Previous literature about students' understanding of heat and temperature primarily emphasizes students' misunderstandings of canonical physics concepts. In our study, we used a resources-oriented approach to analyze 653 student responses to questions about thermal phenomena, looking for ways in which their responses could serve as valuable resources for continued learning. We identified three common conceptual resources: (A) heat transfer is directional; (B) an object's physical properties matter in thermal processes; and (C) hotter objects have more energy. These resources could be used to strengthen physics teaching by using students' understandings of heat and temperature to support the development of more advanced physics ideas. 


\section{INTRODUCTION}

Physics teaching requires pedagogical knowledge (PCK), including knowledge of student ideas (KSI), or what is already known by students about the topic being taught [1]. Previous literature about student understanding of thermodynamics mainly focuses on students' misunderstandings of canonical physics concepts, to understand how to better assist their learning. These misunderstandings are often framed as misconceptions, or ideas that prevent students from answering physics questions correctly $[2,3]$. For example, the literature emphasizes that students often conflate the concepts heat, temperature, and internal energy [4-11]; that students often treat heat as a state, property, or substance $[4,6,9,11-18]$; and that students misapply the ideal gas law and the first law of thermodynamics [4-6,8,9,11,13,19-22].

Our study adds to the literature identifying common student conceptual resources for understanding heat and temperature, which could be used by instructors to build on student ideas about thermodynamics toward more complex understandings $[23,24]$. These are elements of reasoning that recur often in students' answers, are continuous with physics, and are sensible to students and could help them answer questions correctly or logically [25-27].

We identified three common conceptual resources for understanding heat and temperature, based on our analysis of 653 responses to thermodynamics questions: (A) heat transfer is directional; (B) an object's physical properties matter in thermal processes; and (C) hotter objects have more energy. Examples that illustrate how students used these resources in their responses are provided in Section IV.

\section{THEORY}

In resources theory, a resource is a piece of knowledge that gets activated in real time, in context-sensitive ways [3,26-33]. Researchers have theorized extensively about the development, structure, and role of resources, and have used resources theory to highlight the dynamic, emergent, complex-systems-like nature of student thinking.

Our work draws extensively from resource theory's orientation toward student thinking as fundamentally sensible and continuous with physics [3,26,27,29,30,32,33]. Resource theory emphasizes that learners derive resources from their sensory experience and then use these resources to make sense of the material world. Our work seeks to make visible the continuities between students' thinking and formal physics, even and especially when that thinking does not use the language of formal physics or is incorrect. Further, our work builds from resource theory's definition of learning, which involves changing the structure or activation of resources, by reorganizing, refining, increasing the formality of, or changing the role of resources [2629,32,33]. Finally, our work acknowledges that resource activation is context-sensitive $[3,26,28-30,32,33]$ and hypothesizes aspects of context that may shape which resources get activated. Many resources we identify are at the grain size of ideas (rather than, e.g., primitives); this is not inconsistent with resources theory [26].

\section{METHODS}

The resources we report were identified based on our analysis of 653 written student responses to three heat and temperature questions - the chairs question, the big-small blocks question, and the heat transfer question-all shown in Figure 1. All three were constructed for the purposes of this project, with different conceptual targets in mind. The chairs question was meant to target student perceptions of temperature and heat capacity; the big-small blocks question, student thinking about properties of objects in thermal phenomena; and the heat transfer question, mechanisms for energy transfer in thermal phenomena.

There are a variety of correct ways to answer the chairs and heat transfer questions. In the chairs question, one can imagine the exchange of energy from one's body to the chairs and the resulting physical effect-in this case, a change in skin temperature. The difference in perceived temperature can be accounted for by the metal chair's tendency to take energy from one's body faster than a plastic chair, so the metal chair feels colder. In the heat transfer question, one can use either macroscopic or microscopic reasoning. Microscopically, hot objects have higher temperatures, which corresponds to particles with higher kinetic energy. When two objects come into contact, one hot and one cold, the interactions between the higher-and lower-energy particles at the point of contact transfer some of the energy to the colder object.

One correct answer to the big-small blocks question is that temperature changes happen when thermal energy moves into or out of an object. For two objects in contactin this case, a massive block, and water-this thermal energy transfer happens at the point of contact and depends on a multitude of factors including the specific heat capacities of the two objects, the surface area, etc. The more massive block (that is otherwise identical) will have more volume than the small block, with the same energy density. This means that in the big block there are more particles of the same material with the same energy, so the larger block has a greater net energy than the small block. Since the big block has more overall energy than the small block and they lose the same amount of energy, the smaller block loses a greater percentage of its net energy, thus it feels colder.

We analyzed a total of 653 written responses to these three questions from introductory physics courses at four US colleges and universities. U1, U2, and U3 are all large public universities: U1 in the Pacific Northwest US, U2 in the Eastern US, and U3 in the Midwest. U4 is a large public junior college in the Western US. (Sample sizes by question and college/university are given in Table I.) Students 


\begin{tabular}{||l||l||l||}
\hline $\begin{array}{l}\text { Chairs question (Q1): } \\
\text { A plastic chair and a metal } \\
\text { chair that have been sitting in } \\
\text { the same room for a long time } \\
\begin{array}{l}\text { are the same temperature, but } \\
\text { when you sit on the metal } \\
\text { chair, it feels colder. }\end{array}\end{array}$ & $\begin{array}{l}\text { Big-small blocks question (Q2): } \\
\text { Imagine you have two room-temperature blocks made of the same } \\
\text { metal, but one has more mass than the other. You drop the blocks } \\
\text { into equal volumes of 5-degrees-C water, count to five, and then } \\
\text { dump them out onto a table. You measure the temperature of } \\
\text { both blocks, and the less massive one is colder than the more } \\
\text { massive one. That's because the mass of an object matters for } \\
\text { how much its temperature changes. }\end{array}$ & $\begin{array}{l}\text { You may have heard that } \\
\text { "heat" or "thermal energy" } \\
\text { transfers from hot to cold } \\
\text { objects, and not the other way } \\
\text { around. }\end{array}$ \\
$\begin{array}{l}\text { How do you account for this } \\
\text { observation? (If this doesn't } \\
\text { make sense, say why not or } \\
\text { what you expected } \\
\text { differently.) }\end{array}$ & $\begin{array}{l}\text { What we want to know is why that makes sense or doesn't make } \\
\text { sense to you: Why is it that a less massive block changes its } \\
\text { temperature more than a more massive block made of the same } \\
\text { material and at the same starting temperature? }\end{array}$ & $\begin{array}{l}\text { Wow do you make sense of } \\
\text { this phenomenon? }\end{array}$ \\
\hline
\end{tabular}

FIG. 1. Conceptual questions asked in our study.

answered the questions both before and after instruction on homework and quizzes. The course response rates were 79\% for questions asked at $\mathrm{U} 1 ; 31 \%$ for $\mathrm{U} 2 ; 33 \%$ for $\mathrm{U} 3$; and $87 \%$ for U4. Lower course response rates correspond to data collected: at the start of the global COVID-19 pandemic, from group assignments, and in courses with fewer students consenting to participate in research. Each institution selected the question(s) they wanted to ask; not all questions were asked at all institutions.

The racial and/or ethnic demographics for the colleges/ universities in our study versus all college/university students are shown in Figure 2. Figure 2 suggests that the institutions in our study are not racially and/or ethnically representative of the population of college-bound freshmen in the US. The universities in our study serve more Asian and Asian American students, fewer Hispanic or Latinx students, fewer Black or African American students, and fewer white students than are in the general population of college students. In addition, the median parental income of the students at colleges/universities in our study is higher than the national average. This sampling limits the generalizability of our results; though we have documented that the resources we have identified are common among the students in our sample, we cannot speak to the commonality in the population of introductory physics students writ large. One limitation of Figure 2 and our comparison of wealth demographics is that they are based on university-level data, rather than sample-level data. Until we know more about what constitutes a representative sample of introductory physics students, sample-level data does not allow for comparisons to a national average.

We used responses to the questions in Figure 1 to create an emergent coding scheme [34] that included some of the resources that the students in our sample used to reason about heat and temperature. To construct this scheme, authors YA and $\mathrm{MV}$, in consultation with $\mathrm{AR}, \mathrm{LB}$, and $\mathrm{BH}$, conducted preliminary analyses of student responses to each question, looking for ideas that we considered to be continuous with relevant physics concepts, even if not stated in formal terms. We used these preliminary analyses to identify patterns in student reasoning across questions, foregrounding a model of generalizability that emphasizes recurrence across multiple sources of heterogeneity [35]. This process produced a final scheme with three resources.

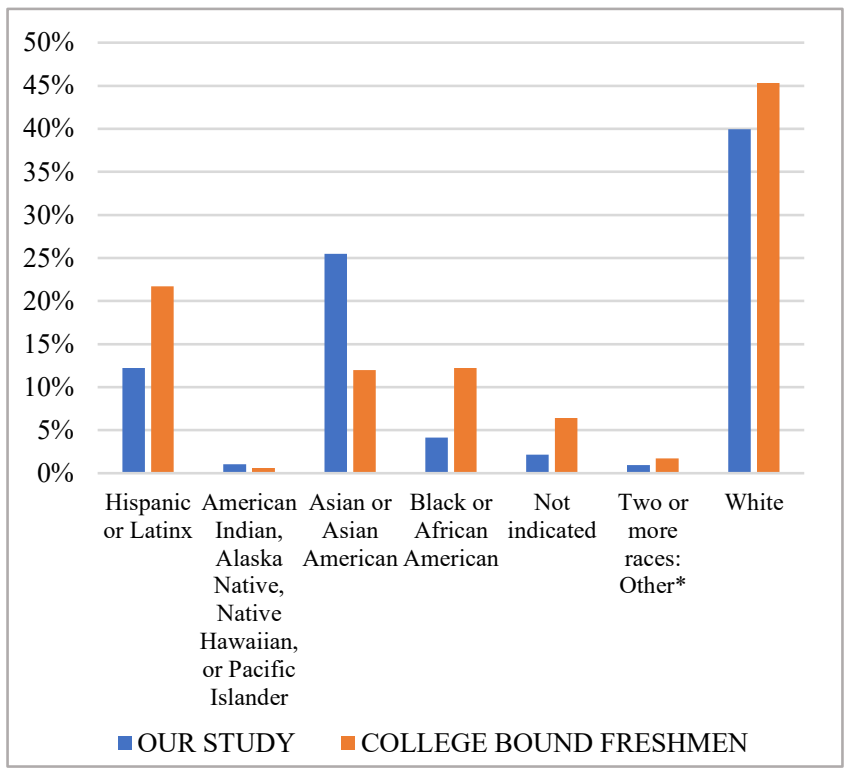

FIG. 2. Racial and/or ethnic demographics of institutions in our sample (blue) versus all college-bound freshmen (orange). Blue bars were constructed using demographic data provided by offices of institutional research or institutional websites, weighted by sample size. Orange bars were constructed using data from Kanim and Cid . [36] As explained by activist Kat Lazo [37], neither Hispanic nor Latinx are racial groups, and these two identities are not the same. "Hispanic" is a descriptor for people of Spanishspeaking origins, and "Latinx" is a descriptor for people with origins in Latin America. The former focuses on language, the latter on geographic location. 
Authors YA and MV independently coded student responses to the three questions. Resources can be-and often are - activated in concert; thus, a single response could receive no code, one code, or many codes. As a measure of inter-rater agreement, we took the normalized difference between the total number of possible codes and the total number of disagreements between the two coders. We used percentage agreement rather than a standard statistical measure of agreement (e.g., Cohen's kappa) because our codes are not necessarily independent or mutually exclusive [38,39]. The percentage agreement for the full data set was $82 \%$.

\section{RESULTS}

In our analysis we identified three common student resources for understanding heat and temperature. These resources had to appear in at least $10 \%$ of students' responses in at least one question, and at some other frequency in additional questions, to be considered common. Table I shows the frequency at which each resource was used in student responses to each question. These resources were used in both correct and incorrect responses.

TABLE I. Prevalence of resource use, by university and question. Responses were only counted if both coders agreed.

\begin{tabular}{lcc|ccc|ccc}
\hline \multicolumn{1}{c|}{} & \multicolumn{9}{c}{ Prevalence } \\
\hline & \multicolumn{2}{c}{ Q1 } & \multicolumn{3}{c|}{ Q2 } & & Q3 \\
& $\mathrm{U1}$ & $\mathrm{U} 2$ & $\mathrm{U} 1$ & $\mathrm{U} 3$ & $\mathrm{U} 4$ & $\mathrm{U} 1$ & $\mathrm{U} 3$ & $\mathrm{U} 4$ \\
$n$ & 135 & 22 & 191 & 18 & 39 & 191 & 18 & 39 \\
\hline $\mathrm{A}$ & $45 \%$ & $27 \%$ & $3 \%$ & $0 \%$ & $3 \%$ & $77 \%$ & $89 \%$ & $97 \%$ \\
$\mathrm{~B}$ & $97 \%$ & $86 \%$ & $92 \%$ & $94 \%$ & $92 \%$ & $0 \%$ & $0 \%$ & $0 \%$ \\
$\mathrm{C}$ & $42 \%$ & $23 \%$ & $9 \%$ & $0 \%$ & $31 \%$ & $21 \%$ & $50 \%$ & $56 \%$ \\
\hline
\end{tabular}

\section{A. Heat transfer is directional.}

Student responses often reflected the idea that energy flows in a specific direction during heat transfer, saying that heat flows from hot to cold, "seeks" thermal equilibrium, or moves from regions of high to low energy. We use the phrase "heat transfer" here to reflect students' reasoning; though heating is a process and not a transferable quantity, we understand students to be using the term "heat" to express a quantity that moves and affects the temperature of objects, akin to thermal energy.

One example of this resource comes from the chairs question: "Though both chairs are at the same temperature, our bodies are at a higher temperature. In order to reach thermal equilibrium between our body and the chair we sit on it, heat from our body must move to [the] chair..." This response not only correctly identifies the direction of heat flow-from the (warmer) body to the (room temperature) chair-but it also names that objects seek thermal equilibrium to explain the directionality of heat transfer.

Some students articulated a mechanism for the directionality of heat transfer, associating temperature and particle motion, such that one object being colder than another one means there is less particle movement. For instance, answering the heat transfer question, one student wrote, "Thermal energy is a result of the movement of the particles so there is no way you could transfer no movement, so the only direction is transferring movement to no movement." Though this student is incorrect in suggesting that colder objects have no particle movement at all, this response does name a mechanism for the directionality of thermal energy transfer: collisions between particles moving at different speeds.

Students often used the "heat transfer is directional" resource in a way that is continuous with the second law of thermodynamics, where heat travels from hot to cold objects. This resource was used most frequently in the heat transfer question (Q3), where it was used by $77 \%, 89 \%$, and $97 \%$ of students in our three samples. It is perhaps not surprising that this resource was used most frequently in the heat transfer question, given that the problem highlights heat flowing from hot to cold objects. However, that so many students used this resource when cued indicates that it was available to them and could be elicited in other contexts.

\section{B. An object's physical properties matter in thermal processes.}

This resource reflects that an object's physical properties, such as mass, material, or state of matter, affect how much its temperature changes or how much thermal energy it transfers. For example, one student answered the chairs question by stating, "Metal chairs feel colder than plastic chairs in the same room because metal is a good conductor of heat while plastics are insulators. Metals allow heat and cold to transfer through them while plastics do not allow heat and cold to transfer through them." Though it is not the case that plastics cannot transfer energy, this student productively identified a difference in thermal properties of different chair materials. Then, the student named a connection between this material difference and the difference in temperature being perceived when the chairs are touched.

In response to the big-small blocks question, one student wrote, "There are fewer molecules that need to have their energy changed for the overall temperature to change." This student approached the question microscopically, referring to a difference in the number of particles in the two blocks. Microscopic responses were more common in the big-small blocks question than in the chairs question.

This resource was $92-94 \%$ and $86-97 \%$ recurrent in the big-small block and chairs questions, respectively. The high frequency of use in these questions makes sense because both questions focus on how the properties of objects affect temperature or heat transfer.

\section{Hotter objects have more energy.}

This resource was coded when a student used reasoning that related the energy of an object to its overall temperature, 
on either a microscopic or macroscopic scale. For example, in the heat transfer question, one student answered, "This makes sense because hot objects have greater kinetic energy. Thus, the atoms of something hot have greater kinetic energy to be able to transfer to something cold." This response draws an explicit connection between the temperature of the object and its energy, reasoning that more kinetic energy in the atoms corresponds to a higher temperature of the block.

Other responses took a macroscopic approach, focusing on the object of interest as a whole and not at a molecular level. For example, in response to the heat transfer question, one student wrote, "Because cold objects are essentially the same thing as hot objects, just with less energy in them. You can only transfer positive energy, not negative." Though this student incorrectly implies that cold objects can have negative energy, they correctly highlight that temperature indicates energy, with hot objects having more energy than cold ones. Both examples are consistent with the broader trend of students associating temperature with energy, and/or treating temperature as an indicator of the object's energy.

The variation in the use of this and the previous resource may have to do with the degree of emphasis of each question on temperature versus properties. The heat transfer question focuses on temperature or heat and not on properties, and this shows up in student use of the resources we identified: resource B (about properties) is not used, whereas resource $\mathrm{C}$ (about the relationship between temperature and energy) is used frequently. The reverse is true of the big-small blocks question, which focuses on properties: resource B shows up a lot, and resource $\mathrm{C}$ very infrequently.

\section{DISCUSSION}

In our study, we identified three conceptual resources for understanding heat and temperature based on our analysis of 653 student responses to questions about thermal phenomena: (A) heat transfer is directional; (B) an object's physical properties matter in thermal processes; and (C) hotter objects have more energy. These resources highlight ways in which student responses appear to be both continuous with formal physics and common among the responses in our sample. Student responses do not need to be correct to draw on these resources: many students marshaled these good ideas in service of incorrect answers.

In identifying these resources, our project expands the previous literature on student thinking about thermal phenomena, which primarily frames students' ideas as misconceptions. Indeed, previous literature highlights that students often conflate the concepts heat, temperature, and internal energy [4-11], or that students incorrectly associate properties such as shape, size, color, thickness, and hardness with temperature change during thermal energy transfer $[7,10,12,15,17]$. Our analysis highlights a number of resources that students use to reason about thermal phenomena. We could think of these three resources as footholds [25], or ideas that can serve as generative starting places for instruction, whereas previous literature frames student misconceptions as barriers or troubling endpoints. For example, our resource B foregrounds that students are associating properties with outcomes in thermal processes, regardless of whether the specific property they name is correct. The literature foregrounds the incorrectness of an association that students are making. In this sense, our work offers a reframing that seeks to focus instructor attention on opportunities to build from students' ideas.

Other misconceptions reported by previous literature are related to students not correctly applying macroscopic and microscopic ideas in thermodynamic scenarios [9]. However, we observed that the way questions are framed might influence students' use of macroscopic or microscopic perspectives. For example, in the chairs question, where there are physically evident differences in the material of the objects, students correctly used macroscopic reasoning to answer the question. However, in questions where there are not evident macroscopic differences in the objects under investigation, like the heat transfer question, students tended to use a microscopic model to answer the question, by emphasizing the amount and the speed or movement of the object's particles. Future research could build on this observation by looking for question-reasoning pairings, identifying which contexts reliably elicit macroscopic versus microscopic reasoning (or both).

Instructors who wish to build on student conceptual resources for understanding heat and temperature could use this research to support in-the-moment listening or instructional planning. Resource $\mathrm{A}$-that heat transfer is directional-is continuous with the second law of thermodynamics. Even when students use this resource to support incorrect reasoning, such as naming a directionality that physics does not endorse, instructors can notice and build from their attribution of directionality to heating. Likewise, instructors can build from students' identification of properties as mattering for temperature change and thermal energy transfer, even when the properties they identify are not part of the canon. Instructors can use what we are learning about context-dependence to choose contexts that elicit an association between temperature and energy, either to refine this association or to apply it to relevant contexts or problems. Though we have identified particular resources and are naming particular ways instructors might build from them, the goal of our work is to illustrate, not prescribe. Our primary aim is to make concrete the general idea that students have wonderful ideas about physics [40], including heat and temperature.

\section{ACKNOWLEDGMENTS}

This work was supported in part by the National Science Foundation under grant numbers 1914603 \& 1914572. The authors wish to thank Lisa Goodhew, Paula Heron, and Rachel Scherr for feedback on drafts of the paper, and all those who helped collect data. 
[1] L. Shulman, Harv. Educ. Rev. 57, (1987).

[2] L. C. McDermott and E. F. Redish, Am. J. Phys. 67, 755 (1999).

[3] D. Brown and D. Hammer, in International Handbook of Research on Conceptual Change, edited by S. Vosniadou (Taylor and Francis, Inc., 2008), p. 127-154.

[4] M. K. Summers, Sch. Sci. Rev. 64, 670 (1983).

[5] J. Clark, J. Thompson, and D. Mountcastle, 1513, 102 (2013).

[6] M. E. Loverude, C. H. Kautz, and P. R. L. Heron, Am. J. Phys. 70, 137 (2002).

[7] H.-E. Chu, D. F. Treagust, S. Yeo, and M. Zadnik, Int. J. Sci. Ed. 34, 1509 (2012).

[8] N. Erceg, I. Aviani, V. Mešić, M. Glunčić, and G. Žauhar, Phys. Rev. Phys. Educ. Res. 12, 020139 (2016).

[9] P. Wattanakasiwich, P. Taleab, M. D. Sharma, and I. D. Johnston, Int. J. of Innovation in Sci. and Math. Ed. 21, 1 (2013).

[10] E. L. Lewis and M. C. Linn, J. of Research in Sci.Teaching 31, 657 (1994).

[11] C. H. Kautz, P. R. L. Heron, M. E. Loverude, and L. C. McDermott, Am. J. Phys. 73, 1055 (2005).

[12] S. Yeo and M. Zadnik, The Phys.Teacher 39, 496 (2001).

[13] P. G. Jasien and G. E. Oberem, J. Chem. Educ. 79, 889 (2002).

[14] M. Arnold and R. Millar, Int. J. Sci. Ed. 16, 405 (1994).

[15] E. E. Clough and R. Driver, Phys. Educ. 20, 176 (1985).

[16] D. Brookes, G. Horton, A. Van Heuvelen, and E. Etkina, AIP Conference Proceedings 790, 149 (2005).

[17] M. Prince, M. Vigeant, and K. Nottis, J. of Eng. Edu. 101, 412 (2012).

[18] G. L. Erickson, Sci. Ed. 63, 221 (1979).

[19] A. D. Robertson and P. S. Shaffer, Am. J. Phys. 81, 303 (2013).

[20] D. E. Meltzer, in Proceedings of Physics Education Research Conference, New York, 2001.
[21] C. H. Kautz, P. R. L. Heron, P. S. Shaffer, and L. C. McDermott, Am. J. Phys. 73, 1064 (2005).

[22] D. E. Meltzer, Am. J. Phy. 72, 1432 (2004).

[23] D. Hammer, F. Goldberg, and S. Fargason, Rev. Sci., Math., and ICT Ed. 6, 1 (2012).

[24] D. Hammer, Cognition Instruct. 15, 485 (1997).

[25] D. Hammer and E. van Zee, Seeing the Science in Children's Thinking: Case Studies of Student Inquiry in Physical Science, 1st edition (Heinemann, Portsmouth, NH, 2006).

[26] D. Hammer, Am. J. Phys. 68, S52 (2000).

[27] J. P. Smith, A. A. diSessa, and J. Roschelle, J. Learn. Sci. 3, 115 (1993).

[28] D. Hammer, A. Elby, R. E. Scherr, and E. F. Redish, in Transfer of Learning from a Modern Multidisciplinary Perspective (IAP, Greenwhich, CT, 2005), p. 89-119.

[29] A. A. diSessa, Cognition Instruct. 10, 105 (1993).

[30] B. Harrer, PhD. thesis, University of Maine, 2013.

[31] R. E. Scherr, Am. J. Physics 75, 272 (2007).

[32] E. Redish, Lecture at Int. Sch. Phys. "Enrico Fermi" Course SLVI, Varenna, Italy (2003).

[33] D. Hammer, J. Learn. Sci. 5, 97 (1996).

[34] K. H. Krippendorff, Content Analysis - 3rd Edition: An Introduction to Its Methodology (SAGE Publications, Inc, Thousand Oaks, 2013).

[35] T. D. Cook, Edu. Eval. and Policy Analy. 24, 175 (2002).

[36] S. Kanim and X. C. Cid, Phys. Rev. Phys. Educ. Res. 16, 020106 (2020).

[37] V. M. Massie, Retrieved: https://www.vox.com/2016/8/28/12658908/latin o-hispanic-race-ethnicity-explained.

[38] M. L. McHugh, Biochem Med (Zagreb) 22, 276 (2012).

[39] J. Cohen, Edu.Psych. Meas. 20, 37 (1960).

[40] E. Duckworth, The Having of Wonderful Ideas" and Other Essays on Teaching and Learning, 3rd edition (Teachers College Press, New York, 2006). 